\title{
Emergent White Matter Degeneration in the rTg-DI Rat Model of Cerebral Amyloid Angiopathy Exhibits Unique Proteomic Changes
}

Joseph M. Schrader, ${ }^{* \dagger}$ Feng Xu,$^{* \dagger}$ Hedok Lee, ${ }^{\ddagger}$ Benjamin Barlock, ${ }^{\dagger}$ Helene Benveniste, ${ }^{\ddagger}$ and William E. Van Nostrand ${ }^{* \dagger}$

From the George \& Anne Ryan Institute for Neuroscience* and Department of Biomedical and Pharmaceutical Sciences, ${ }^{\dagger}$ University of Rhode Island, Kingston, Rhode Island; and the Department of Anesthesiology, ${ }^{\ddagger}$ Yale University, New Haven, Connecticut

Accepted for publication November 23, 2021.

Address correspondence to William E. Van Nostrand, Ph.D., Department of Biomedical and Pharmaceutical Sciences, George and Anne Ryan Institute for Neuroscience, University of Rhode Island, 130 Flagg Rd., Kingston, RI 02881. E-mail: wvannostrand@uri.edu.

\begin{abstract}
Cerebral amyloid angiopathy (CAA), characterized by cerebral vascular amyloid accumulation, neuroinflammation, microbleeds, and white matter (WM) degeneration, is a common comorbidity in Alzheimer disease and a prominent contributor to vascular cognitive impairment and dementia. WM loss was recently reported in the corpus callosum (CC) in the rTg-DI rat model of CAA. The current study shows that the CC exhibits a much lower CAA burden compared with the adjacent cortex. Sequential Window Acquisition of All Theoretical Mass Spectra tandem mass spectrometry was used to show specific proteomic changes in the $\mathrm{CC}$ with emerging WM loss and compare them with the proteome of adjacent cortical tissue in $\mathrm{rTg}$-DI rats. In the $\mathrm{CC}$, annexin $\mathrm{A} 3$, heat shock protein $\beta 1$, and cystatin $C$ were elevated at 4 months (M) before WM loss and at $12 \mathrm{M}$ with evident WM loss. Although annexin $\mathrm{A} 3$ and cystatin $C$ were also enhanced in the cortex at $12 \mathrm{M}$, annexin $\mathrm{A} 5$ and the leukodystrophy-associated astrocyte proteins megalencephalic leukoencephalopathy with subcortical cysts 1 and GlialCAM were distinctly elevated in the CC. Pathway analysis indicated neurodegeneration of axons, reflected by reduced expression of myelin and neurofilament proteins, was common to the $\mathrm{CC}$ and cortex; activation of Tgf$\beta 1$ and F2/thrombin was restricted to the CC. This study provides new insights into the proteomic changes that accompany WM loss in the CC of rTg-DI rats. (Am J Pathol 2022, 192: 426-440; https:// doi.org/10.1016/j.ajpath.2021.11.010)
\end{abstract}

Cerebral amyloid angiopathy (CAA), a disease characterized by accumulation of fibrillar amyloid $\beta$-protein $(\mathrm{A} \beta)$ in blood vessels of the brain, is prevalent in the elderly population and is a common comorbidity of Alzheimer disease. ${ }^{1-4}$ There are two major forms of CAA: CAA type 1, in which amyloid accumulates along cerebral microvessels and capillaries, and CAA type 2 , in which the $A \beta$ deposition occurs within the walls of small arteries and arterioles. ${ }^{5}$ CAA can promote degeneration of mural and endothelial cells of the vessel wall, leading to cerebral microbleeds and larger hemorrhages, as well as thrombotic occlusions resulting in ischemic lesions, which collectively can cause white matter (WM) damage and vascular cognitive impairment and dementia. ${ }^{1,2,6-8}$ In addition to the apparent loss of myelin proteins, other changes that occur in the proteome of the WM in conjunction with CAA are not well understood and may provide further insight into the pathogenesis of this region.
Prior studies focused on the generation and characterization of a transgenic rat model of CAA type 1 known as rTg-DI. ${ }^{9}$ The rTg-DI rats produce human $\mathrm{A} \beta$ harboring the Dutch (E22Q) and Iowa (D23N) familial CAA mutations in the brain and develop early-onset accumulation of cerebral microvascular amyloid in the absence of parenchymal plaque amyloid deposits. The progressive accumulation of microvascular CAA in this model promotes robust perivascular neuroinflammation, degeneration of pericytes, thalamic cerebral microbleeds and occluded microvessels, and behavioral impairments reminiscent of "cognitive slowing." ${ }^{9-12}$ Recently, rTg-DI rats were shown to present

Supported by NIH research grant RO1NS094201 (W.E.V.N.) and RF-AG053991 (H.B., W.E.V.N.).

Disclosures: None declared.

Current address of B.B., Merck \& Co., Boston, MA. 
with diffuse WM loss emerging after 6 months (M) and progressing to up to $12 \mathrm{M} .{ }^{13}$ The WM loss was primarily observed in the corpus callosum (CC) and in the globus pallidus and thalamus, as detected by magnetic resonance imaging. Despite the clear loss of WM, an understanding of how cerebral microvascular amyloid accumulation in aging rTg-DI rats affects the CC is unclear.

Liquid chromatography coupled with tandem mass spectrometry (MS/MS) has provided a means for reliable and sensitive high-throughput proteomic investigation. ${ }^{14,15}$ Data-independent acquisition approaches, such as Sequential Window Acquisition of All Theoretical Mass Spectra (SWATH-MS), which perform quantitative analysis of all detected protein fragments and characterize thousands of proteins within a single sample, are becoming the standard for cohort proteomics. ${ }^{14,16,17}$ Recently, brain regional proteomic alterations were reported in the cortex, hippocampus, and thalamus of $12 \mathrm{M}$ rTg-DI rats using laser microdissection and SWATH-MS. ${ }^{12}$ Specific proteomic changes with regionally distinct pathologies were used to describe important mechanisms implicated in the development of the differential pathologic phenotypes.

The current study uses laser microdissection and SWATH-MS to investigate the role of progressive CAA type 1 in $\mathrm{rTg}$-DI rats in changing the protein profile of the CC that exhibits prominent WM loss. These proteomic changes were compared with recent findings in the cortex of $12 \mathrm{M} \mathrm{rTg-DI}$ rats. $^{12}$ Following the proteomics, bioinformatics analysis was conducted using Ingenuity Pathway Analysis (IPA) (Qiagen Inc., Redwood City, CA) and brain tissue immunolabeling to connect the proteomic alterations with specific pathways and cellular responses implicated in the progressive WM loss.

\section{Materials and Methods}

Animals

The rTg-DI transgenic rat model of CAA was generated as previously described $^{9}$ in which low levels of human Swedish/Dutch/Iowa mutant A $\beta$ protein precursor are expressed in neurons under the control of the Thy 1.2 promoter and maintained on a Sprague-Dawley background. In this model, the accumulation of microvascular amyloid begins at approximately $3 \mathrm{M}$ and progresses with advancing age. In the current study, three male and three female heterozygous rTg-DI rats and nontransgenic, littermate wildtype (WT) rats were collected at $4 \mathrm{M}$, and three male and two female heterozygous rTg-DI rats and two male and three female WT rats were collected at $12 \mathrm{M}$. The study was neither randomized nor blinded.

All rats were housed in a controlled room $\left(22 \pm 2^{\circ} \mathrm{C}\right.$ and $40 \%$ to $60 \%$ humidity) on a standard 12-hour light cycle. Rat chow and water were available ad libitum. All work with animals was approved by the University of Rhode Island and Yale University Institutional Animal Care and Use
Committees, in accordance with the United States Public Health Service's Policy on Humane Care and Use of Laboratory Animals, and in compliance with the Animal Research: Reporting of In Vivo Experiments guidelines.

\section{Magnetic Resonance Imaging of WM Loss}

The composite data presented were previously acquired from Lee et al. ${ }^{13}$ Briefly, WT and rTg-DI rats $(n=11$ per group) anesthetized with dexmedetomidine and low-dose isoflurane underwent proton density-weighted imaging and were analyzed by using voxel-based morphometry in studying whole-brain-wide WM morphometry as previously described. ${ }^{13,18,19}$ Spatial registration parameters between tissue probability maps and individual scans were calculated by using the DARTEL algorithm (Diffeomorphic Anatomical Registration Through Exponentiated Lie Algebra) and subsequently smoothed by a 0.6 -mm Gaussian smoothing kernel. The resulting smoothed and normalized WM density images were population averaged for the WT and rTg-DI cohorts and overlaid on the corresponding proton density-weighted anatomical template using PMOD version 3.807 software (PMOD Technologies GmbH, Zurich, Switzerland). Two-dimensional displays at the level of the $\mathrm{CC}$ and striatum were chosen for the comparisons of WM volume across ages and strains. Additional details are provided in Lee et al. ${ }^{13}$

\section{Brain Tissue Collection and Preparation}

At the designated ages, anesthetized rats were transcardially perfused with phosphate-buffered saline. The rat brain was surgically removed and bisected in the mid-sagittal plane. One hemisphere was fixed in $4 \%$ paraformaldehyde for immunohistochemical analysis, and the other hemisphere was snap-frozen in optimal cutting temperature medium (OCT 4585, Fisher Healthcare, Waltham, MA) directly.

\section{Protein Digest of Collected CC Tissue}

The CC region from brain sections of WT and rTg-DI rats was collected by using laser capture microdissection. Briefly, 20 sagittal sections $(25 \mu \mathrm{mol} / \mathrm{L}$ thick) of fresh frozen $4 \mathrm{M}$ and $12 \mathrm{M}$ rat brain hemispheres were prepared on laser microdissection framed membrane slides (Leica Microsystems, Buffalo Grove, IL), two sections per slide. The CC from each tissue section was collected by using laser capture microdissection and pooled for each rat. Tissue lysis and sample preparation for MS analysis were performed as previously described. ${ }^{12}$ Briefly, tissue was lysed in radioimmunoprecipitation assay buffer via sonication. Protein was denatured by adding $25 \mu \mathrm{L}$ dithiothreitol (100 $\mathrm{mmol} / \mathrm{L}$ ) and incubation with shaking (300 rpm) at $95^{\circ} \mathrm{C}$ for 15 minutes, and samples were alkylated with $25 \mu \mathrm{L}$ iodoacetamide $(200 \mathrm{mmol} / \mathrm{L})$ and incubated in the dark for 30 minutes at $20^{\circ} \mathrm{C}$. Protein was then precipitated and 
concentrated via chloroform-methanol-water (1:2:1) precipitation and washed with methanol. Samples were resuspended in sodium deoxycholate $(3 \% \mathrm{w} / \mathrm{v}$ in $50 \mathrm{mmol} / \mathrm{L}$ ammonium bicarbonate). Proteins were digested with tosylsulfonyl phenylalanyl chloromethyl ketone (TPCK)treated trypsin at a concentration of 1:20 trypsin:protein (Sciex, Framingham, MA) in a barocycler (Pressure Biosciences Inc., Easton, MA) as previously described. ${ }^{12}$ Deoxycholate was precipitated by the addition of formic acid $(5 \% \mathrm{v} / \mathrm{v}$, in $50 \% \mathrm{v} / \mathrm{v}$ acetonitrile in water) for a final concentration of $0.5 \% \mathrm{v} / \mathrm{v}$, followed by centrifugation $(15,300 \times g, 5$ minutes, at room temperature $)$, and the supernatant was collected for liquid chromatography-MS/MS analysis.

\section{Analysis by Liquid Chromatography Quadrupole Time-of-Flight/MS}

Proteomic experiments were performed on a SCIEX 5600 TripleTOF mass spectrometer in positive ion mode using a DuoSpray ion source (AB Sciex, Concord, ON, Canada), coupled to an Acquity UPLC H-Class system (Waters Corp., Milford, MA) for chromatographic separation as previously described. ${ }^{12}$ Peptide separation was achieved by using an Acquity UPLC Peptide BEH $\mathrm{C}_{18}$ column $(2.1 \times 150 \mathrm{~mm}, 300 \AA, 1.7 \mu \mathrm{m})$ preceded by an Acquity VanGuard pre-column $(2.1 \times 5 \mathrm{~mm}, 300 \AA, 1.7 \mu \mathrm{m})$ according to the previously described method. ${ }^{12}$ Trypsindigested $\beta$-galactosidase was injected every four samples to monitor time-of-flight mass calibration. Data were acquired by using Analyst TF 1.7.1 software (AB Sciex) in data-independent acquisition mode. For all dataindependent acquisition experiments, MS settings were exactly as previously described. ${ }^{12}$

\section{Data Processing}

Proteins were identified and quantified by using Spectronaut (release version 13.919110643655, 2019; Biognosys, Schlieren, Switzerland), referencing our previously formed spectral library. ${ }^{12}$ All Spectronaut settings were factory default, except that "used Biognosys' iRT kit" and "PTM localization" were deselected. After Spectronaut analysis, two samples (one 12M WT and one 12M rTg-DI) were removed from subsequent analysis because the number of proteins identified in those samples was substantially lower ( $<60 \%$ of all identified proteins). Raw protein intensities from Spectronaut were then converted to molar concentrations (picomoles per milligram total protein) using the total protein approach. ${ }^{20,21}$ For protein quantities of zero in individual samples, we imputed a baseline concentration (0.03 $\mathrm{pmol} / \mathrm{mg}$ tissue) determined as previously described. ${ }^{12}$ Differentially expressed proteins were defined as $\geq 50 \%$ increase or $\geq 30 \%$ decrease, and statistical significance $(P<0.05)$ was determined via the $t$-test in Excel (Microsoft Corporation, Redmond, WA).

\section{Immunohistochemical Analysis}

Sections were cut from frozen paraformaldehyde-fixed brain hemispheres in the sagittal plane at $20 \mu \mathrm{m}$ thickness using a cryostat (Leica Microsystems) and placed on slides. Antigen retrieval was conducted via 5 minutes' incubation with proteinase $\mathrm{K}(0.2 \mathrm{mg} / \mathrm{mL})$ at $22^{\circ} \mathrm{C}$. Tissue sections were then blocked in SuperBlock blocking buffer (37518; Thermo Fisher Scientific, Waltham, MA) containing 0.3\% Triton X-100 at room temperature for 30 minutes. They were then incubated with individual primary antibodies at the following dilutions overnight: rabbit polyclonal antibody to collagen IV to identify cerebral blood vessels (1:250, SD2365885; Invitrogen); goat polyclonal antibodies to glial fibrillary acidic protein (GFAP; 1:250, ab53554, Abcam) or ionized calcium-binding adapter molecule 1 (Iba-1; 1:250, NB100-1028, Novus) were used to identify astrocytes and microglia, respectively; and rabbit polyclonal antibodies to annexin 5 (1:250, PA578784, Invitrogen), annexin A3 (Anxa3; 1:250, PA5082483, Invitrogen), megalencephalic leukoencephalopathy with subcortical cysts 1 (MLC1; 1:250, PA541042, Invitrogen), and GlialCAM (1:250, PA599999, Invitrogen). The primary antibodies were detected with Alexa Fluor 594- or 488-conjugated secondary antibodies (1:1000). Deposits of fibrillar vascular amyloid were detected by staining with either thioflavin $S$ (123H0598, Sigma Aldrich, St. Louis, MO) or Amylo-Glo (TR-300-AG, Biosensis Inc., Thebarton, SA, Australia) as described by the manufacturer. Myelin was stained and visualized by using the Black Gold II Myelin Staining Kit according to the manufacturer's protocol (AG105; MilliporeSigma, Burlington, MA). Immunohistologic images were captured on a Keyence BZ-X710 fluorescence microscope and analyzed with BZ-X Analyzer software version 1.3.1.1 (Keyence, Itasca, IL).

\section{Data Availability}

Raw MS data can be found in the MassIVE repository (project ID\# MSV000088077; password: rtgdi2021cc).

\section{Results}

\section{Age-Dependent WM Loss in the CC of rTg-DI Rats}

rTg-DI rats were recently shown to exhibit significant WM degeneration compared with WT controls as they age from $6 \mathrm{M}$ to approximately $12 \mathrm{M}$ with advancing microvascular CAA burden. ${ }^{13}$ A composite image of this analysis of the WM loss is shown in Figure 1. In the WT cohort, WM volume increased in the CC (as well as in the septum) from $3 \mathrm{M}$ to $12 \mathrm{M}$ secondary to normal brain maturation and growth. In contrast, in the rTg-DI cohort, the normal growth trajectory of WM volume from $3 \mathrm{M}$ to $12 \mathrm{M}$ at the level of the CC was not evident due to WM loss secondary to the progression of extensive CAA pathologies. The subsequent 


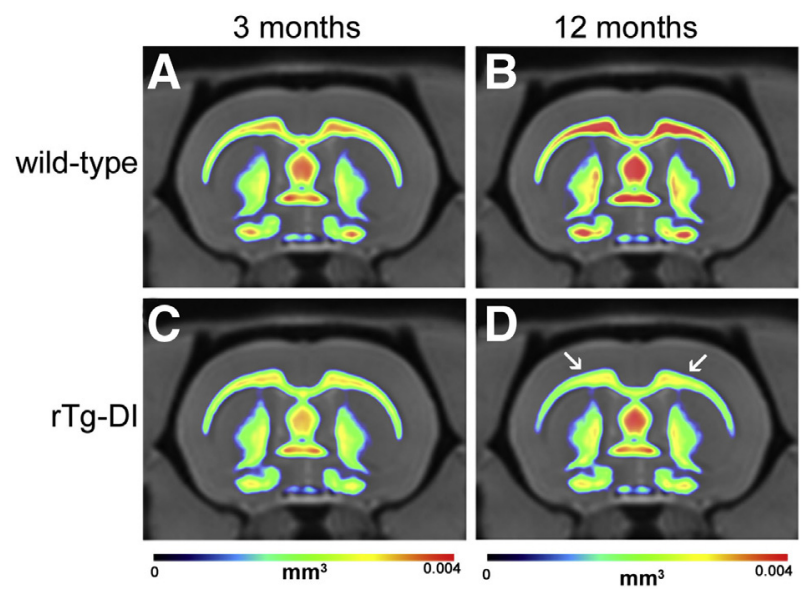

Figure 1 Significant white matter (WM) loss in the corpus callosum (CC) is evident in aged rTg-DI rats. Color-coded population-averaged WM volumetric maps at the level of the CC from a cohort of WT rats scanned at 3 months (3M) (A) and again at approximately 12 months (12M) (B) of age. Corresponding color-coded WM volumetric maps from a cohort of rTg-DI rats scanned at similar $3 \mathrm{M}$ (C) and approximately $12 \mathrm{M}$ (D) time points overlaid on the anatomical template. The color-coded map represents WM volume in each voxel, and blue and red colors indicate high and low WM volume, respectively. In the WT cohort, WM volume increased in the CC (as well as in the septum) from $3 \mathrm{M}$ to $12 \mathrm{M}$ secondary to normal brain maturation and growth. In contrast, in the rTg-DI cohort, the normal growth trajectory of WM volume from $3 \mathrm{M}$ to $12 \mathrm{M}$, at the level of the $\mathrm{CC}$, is not evident due to WM loss secondary to cerebral amyloid angiopathy pathology. The arrows in $\mathbf{D}$ identify the CC exhibiting WM loss. $n=11$.

studies were designed to further investigate the loss of WM in the $\mathrm{CC}$ of aging rTg-DI rats.

\section{rTg-DI Rats Do Not Exhibit Robust Microvascular Amyloid Accumulation in the CC}

Because the magnetic resonance imaging studies presented in Figure 1 indicate the prominent loss of WM in the CC of rTg-DI rats, the presence of CAA was first evaluated in this region and the adjacent cortical area. Prior studies showed that rTg-DI rats develop cerebral microvascular fibrillar amyloid in regions including the cortex, hippocampus, and thalamus, starting at $3 \mathrm{M}$, and sharply increase with age. ${ }^{9,10}$ At $4 \mathrm{M}$, very low levels of microvascular CAA (percent capillary area coverage) were present in both the cortex and CC but were still significantly higher in the cortex (Figure 2, $\mathrm{A}, \mathrm{C}$, and E). However, as rTg-DI rats aged to $12 \mathrm{M}$, the levels of vascular amyloid rose in the cortex to nearly $30 \%$ (Figure 2, B and F). In contrast, the adjacent $\mathrm{CC}$ region continued to exhibit scant amyloid capillary coverage (Figure 2, D and F). These findings indicate that the CC region develops little CAA accumulation compared with the adjacent cortical regions.

Proteomic profiling was performed to further understand the basis for the pronounced WM degeneration in the CC of aging rTg-DI rats. Brain tissue sections from rTg-DI and WT rats at $4 \mathrm{M}$ to $12 \mathrm{M}$ were stained for myelin, and the $\mathrm{CC}$ was collected by using laser capture microdissection.
Supplemental Figure S1 displays representative images of brain sections that were stained for myelin to identify the $\mathrm{CC}$ region that was subsequently microdissected and collected from each rat. Similar to the magnetic resonance images shown in Figure 1, the CC regions of 4M WT and rTg-DI rats were comparable (Supplemental Figure S1, A and $\mathrm{C}$, respectively), whereas at $12 \mathrm{M}$, there was a notable reduction in this region in the rTg-DI rats compared with WT rats (Supplemental Figure S1, B and D) as previously reported. $^{13}$

\section{Enhanced Proteins in $12 \mathrm{M}$ and $4 \mathrm{M}$ rTg-DI Rat CC}

Proteomic analysis of the laser capture microdissection collected CC sections was performed with a dataindependent acquisition liquid chromatography-MS/MS approach as described recently ${ }^{12}$ and in the Materials and Methods. Average molar concentrations from the rTg-DI and WT rats were compared at $4 \mathrm{M}$ and $12 \mathrm{M}$ according to the "total protein approach." 20 Because multiple testing-corrected false discovery rates are often too
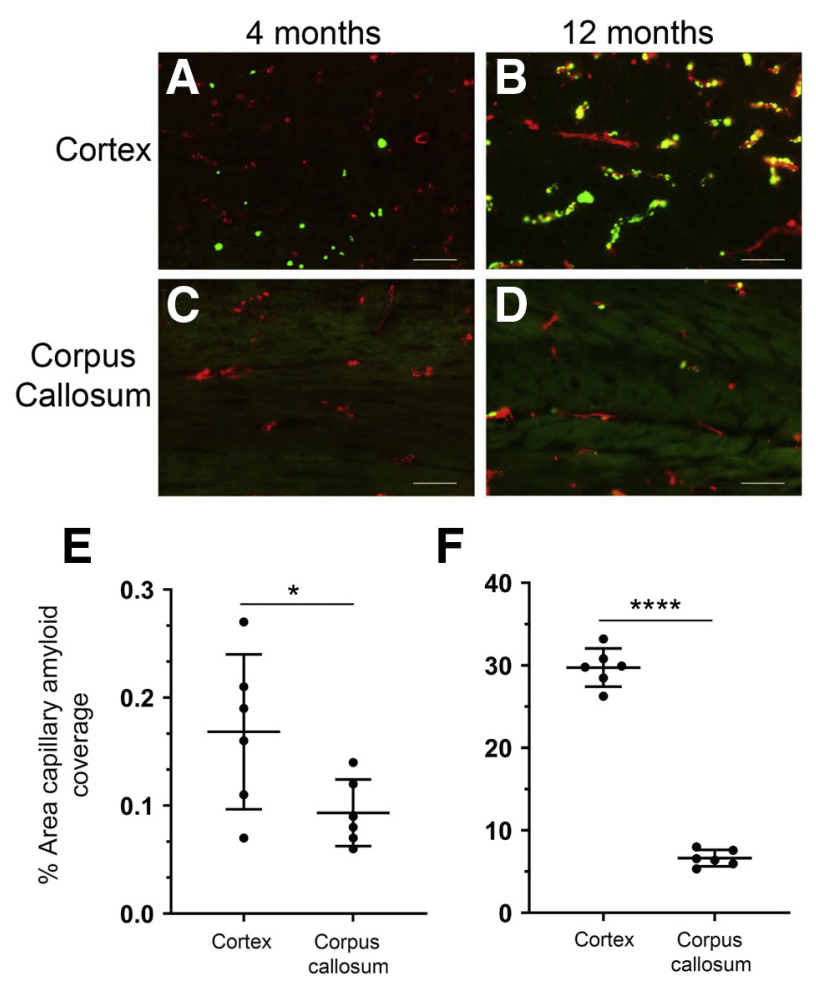

$\mathbf{F}$

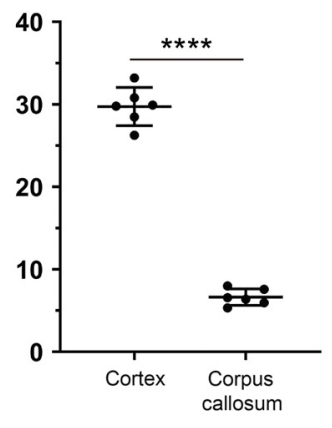

Figure 2 Progressive accumulation of cerebral microvascular amyloid in the cortex and corpus callosum (CC) of rTg-DI rat brain. Brain sections from rTg-DI rats at 4 months (4M) to 12 months (12M) were immunolabeled with rabbit polyclonal antibody to collagen IV to specifically detect cerebral microvessels (red) and stained with thioflavin $\mathrm{S}$ to identify fibrillar amyloid (green). The cerebral microvascular fibrillar amyloid deposits in the cortex and $C$ C at $4 M$ (A and $\mathbf{C}$, respectively) and 12M (B and D). Quantitation of cerebral microvascular amyloid load in the cortex and CC of rTg-DI rats at $4 M(E)$ and $12 M(\mathbf{F})$. Data points show the results from each rat and the group mean \pm SD of $n=6 \mathrm{rTg}$-DI rats at each age. ${ }^{*} P<0.05$; ${ }^{* * *} P<0.0001$. Scale bars $=50 \mu \mathrm{m}(\mathbf{A}-\mathbf{D})$. 
restrictive in small $n$ proteomic studies, ${ }^{22,23}$ uncorrected $P$ values were used for differentially expressed proteins and effect threshold cutoffs were used to manage false discovery rates as previously described. ${ }^{12}$ Nineteen and 73 significantly enhanced proteins $(\geq 50 \%$ increase over WT; $P<0.05)$ were identified in the $4 \mathrm{M}$ and $12 \mathrm{M}$ rTg-DI CC, respectively. These proteins are listed in Supplemental Tables S1 and S2. A Venn diagram comparison of the enhanced proteins at each age is displayed in Figure 3A. Of these, only six proteins, cystatin $\mathrm{C}$, heat shock protein $\beta 1$ (HspB1), Anxa3, proteosome activator complex subunit 1,
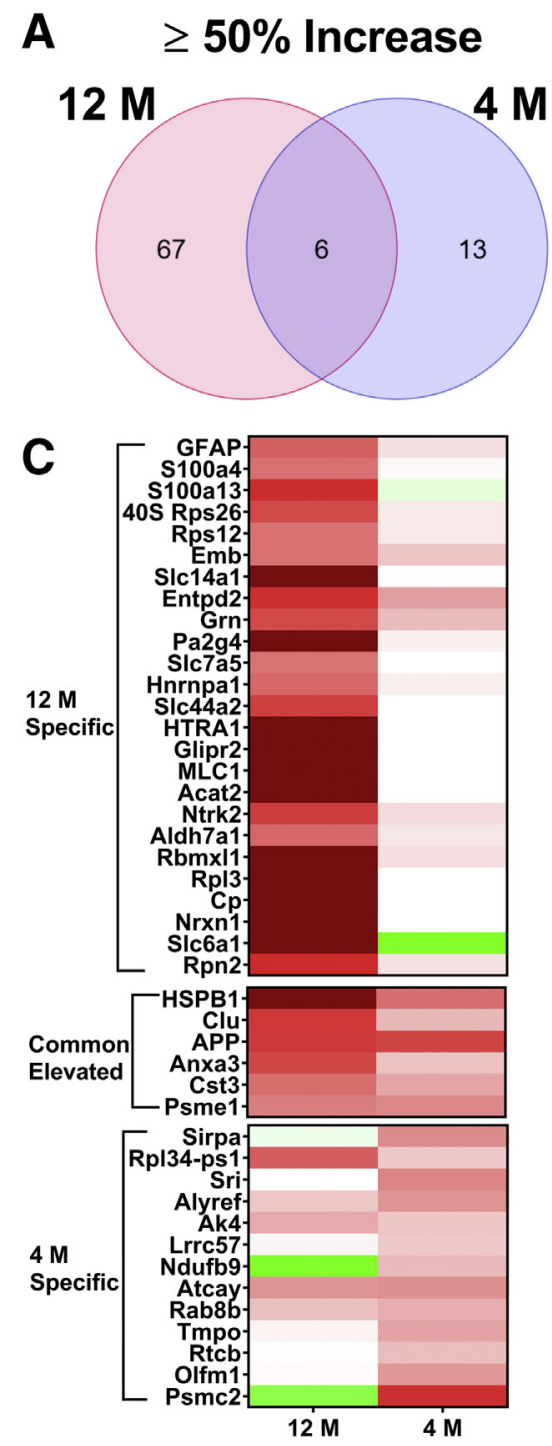

Figure 3 Significantly enhanced and reduced proteins in the rTg-DI corpus callosum (CC) at 12 months (12M) and 4 months (4M). A: Venn diagram comparing significantly enhanced proteins by $\geq 50 \%$ of the wild-type concentration in the CC of rTg-DI rats at $12 \mathrm{M}$ and $4 \mathrm{M}$. B: Venn diagram comparing significantly reduced proteins by $\geq 30 \%$ of the wild-type concentration in the CC of rTg-DI rats at 12M and 4M. C: Heat map depicting relative expression of most enhanced proteins at 12M only (top), at both 12M and 4M (middle), and at 4M only (bottom), along with the corresponding expression in the other age group, with red indicating enhanced expression, green representing reduced expression, and color intensity correlating with degree of change. $\mathbf{D}$ : Heat map depicting relative expression of most reduced proteins arranged as in $\mathbf{C}$ with color shading as in $\mathbf{C} . n=5,6(\mathbf{A}$ and $\mathbf{B})$. $P<0.05(\mathbf{A}$ and $\mathbf{B})$. Anxa3, annexin $\mathbf{A} 3$; ApoE, apolipoprotein E; App, amyloid precursor protein; Clu, clusterin; Cp, ceruloplasmin; Cst3, cystatin C; GFAP, glial fibrillary acidic protein; HTRA1, hightemperature requirement A serine peptidase 1 ; HSPB1, heat shock protein $\beta 1 ; \mathrm{Krt8}$, keratin 8; Mag, myelin-associated glycoprotein; Mbp, myelin basic protein; MLC1, megalencephalic leukoencephalopathy with subcortical cysts 1; Mog, myelin-oligodendrocyte glycoprotein; Nefh, neurofilament heavy chain; Nefl, neurofilament light polypeptide; Nefm, neurofilament medium polypeptide; Plp1, proteolipid protein; Psme1, proteosome activator complex subunit 1; S100a4, S100 calcium binding protein A4; S100A13, S100 calcium binding protein A13; Slc14a1, solute carrier family 14 member 1. 
S100 calcium binding protein A4, high-temperature requirement A serine peptidase 1 (HTRA1), GFAP, MLC1, and ceruloplasmin, as enhanced expression of these proteins was recently reported in other brain regions of $12 \mathrm{M}$ rTg-DI rats. ${ }^{12}$ Thus, despite a relatively low CAA burden, the rTg-DI CC displays enhancement of multiple proteins in common with other rTg-DI brain regions with a much higher CAA burden. Thirteen proteins were elevated in the $4 \mathrm{M}$ rTg-DI CC that were not at $12 \mathrm{M}$, and relative expression of these proteins is similarly depicted in Figure 3C. Of these proteins, only thymopoietin was previously reported as enhanced in other brain regions of rTg-DI rats. However, the six proteins commonly enhanced in the rTg-DI CC at $4 \mathrm{M}$ and $12 \mathrm{M}$ were among those reported as enhanced in other regions of rTg-DI rats. Therefore, before emergence of robust CAA pathology in other brain regions, many of the rTg-DI specifically enhanced proteins are already enhanced at $4 \mathrm{M}$ in the CC. Interestingly, despite the lack of robust CAA in the rTg-DI CC (Figure 2), significant increases in GFAP, a common astrocyte marker, were observed in both the $12 \mathrm{M}$ and $4 \mathrm{M} \mathrm{rTg-DI} \mathrm{CC.}{ }^{24-26}$

\section{Decreased Proteins in $12 \mathrm{M}$ and $4 \mathrm{M}$ rTg-DI Rat CC}

Fourteen and 10 significantly decreased proteins $(\geq 30 \%$ decrease, WT expression $\geq 2 \mathrm{pmol} / \mathrm{mg} ; P<0.05$ ) were identified in the $12 \mathrm{M}$ and $4 \mathrm{M} \mathrm{rTg}-\mathrm{DI} \mathrm{CC}$, respectively, and are listed in Supplemental Tables S3 and S4. A Venn diagram comparing the significantly down-regulated proteins at each age is depicted in Figure 3B, revealing no proteins shared between the two age groups. Expression of these proteins relative to WT are depicted in the heat map in Figure 3D, alongside corresponding relative expression from the other rTg-DI CC age group. Of particular interest in this study are the significant reductions in the myelin related proteins and the neurofilament proteins. For example, myelin basic protein (Mbp) was reduced by $30 \%$ (one-tailed $t$-test, $P=0.033$ ), consistent with recent reported findings via quantitative immunoblotting. ${ }^{13}$ Dysregulation of neurofilament proteins is a hallmark of neurodegenerative diseases, ${ }^{27}$ whereas myelin-associated glycoprotein (Mag), Mbp, and myelin proteolipid protein (Plp1) are all important for axonal myelination, and reductions in their expression could lead to demyelination and disrupted axonal integrity. ${ }^{28-30}$ These results are consistent with the previously reported age-dependent WM degeneration, ${ }^{13}$ and shown in Figure 1, as these dramatic decreases in protein expression are only seen in the $12 \mathrm{M}$ rTg-DI CC.

\section{Differentially Expressed CC Proteins Compared with Those in Cortex}

Regional pathologic characteristics and proteomic changes in the cortex, hippocampus, and thalamus of rTg-DI rats were reported recently. ${ }^{12}$ Although neuroinflammation was present in each region, CAA load in the cortex was moderate compared with higher levels of CAA present in the other two regions. Also, pathway analysis revealed that neurodegeneration of axons was implicated both in the cortex and hippocampus of rTg-DI rats. ${ }^{12}$ The dramatic loss of WM (Figure 1) and the differential expression of proteins associated with neurodegeneration despite minimal CAA load in rTg-DI CC could indicate an impact of the pathology of neighboring regions on the CC. For example, Wallerian degeneration, or the progressive degeneration of distal portions of an injured axon, ${ }^{31}$ could account for degeneration of axons in the $\mathrm{CC}$ of nerves originating and damaged in other brain regions. Therefore, the enhanced proteins in the CC were compared with those in the cortex of $12 \mathrm{M} \mathrm{rTg}$ DI rats, as these neighboring regions share characteristics of neurodegeneration, whereas the higher CAA load is restricted to the cortex. Of the 73 significantly enhanced proteins in the $\mathrm{CC}, 18$ were also elevated in the cortex (Figure 4A). Fifty-five proteins were uniquely elevated in the $\mathrm{CC}$, whereas 42 proteins were elevated in the cortex, but not in the CC.

These proteins were ranked according to increased expression over WT, and the relative expression for the top 25 proteins uniquely enhanced in the CC and in the cortex, along with all proteins commonly enhanced in those regions, is depicted in a heat map in Figure 4C. Of the commonly elevated proteins, GFAP, S100 calcium binding protein $\mathrm{A} 4$, apolipoprotein $\mathrm{E}$, keratin 8 , and amyloid precursor protein were more robustly increased in the cortex and could reflect responses to the higher CAA burden in this region. However, in the CC, S100 calcium binding protein A13, solute carrier family 14 member 1 , and ceruloplasmin were more strongly elevated than in the cortex and could reflect responses unique to this region. Also of note is the dramatic increase in HspB1 observed in the CC. Significantly increased HspB1 expression was recently reported in the hippocampus and thalamus but not in the cortex of $\mathrm{rTg}$ DI rats. ${ }^{12}$ Here in the CC, HspB1 represents a $>500 \%$ increase over WT expression. In addition, the common enhancement of HTRA1 in the CC and cortex is intriguing. Proteomic analysis in human CAA cases has also revealed elevated expression of HTRA $1 .{ }^{22}$ Furthermore, mutations in the HTRA1 gene and loss of HTRA1 function cause cerebral autosomal recessive arteriopathy with subcortical infarcts and leukoencephalopathy, an inherited non-amyloidal cerebral small vessel disease also associated with intracerebral hemorrhage and vascular cognitive impairment and dementia. ${ }^{32-35}$ Therefore, the increases in HTRA1 observed here are noteworthy, as is the potential impact on CAA pathologies in the rTg-DI model.

The two regions displayed less commonality in proteins with significantly reduced expression. Six proteins were reduced in both regions (Figure 4B). A heat map comparing the relative expression of proteins specifically decreased in the $\mathrm{CC}$ or the cortex, and those common to both regions, is displayed in Figure 4D. Commonly decreased proteins 

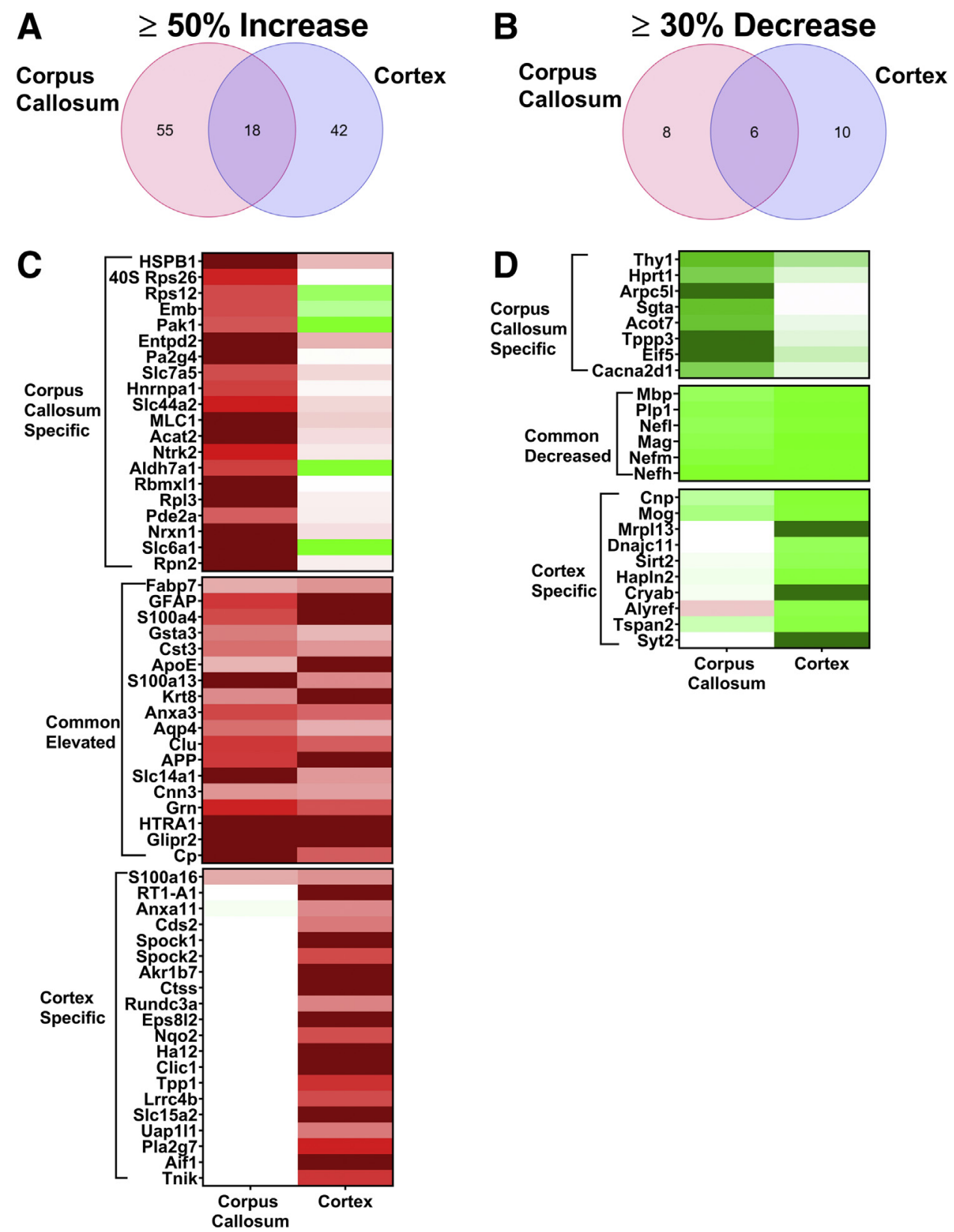

Figure 4 Comparison of significantly enhanced and reduced proteins in the rTg-DI corpus callosum (CC) and cortex at 12 months (12M). A: Venn diagram comparing significantly enhanced proteins by $\geq 50 \%$ of the wild-type concentration in the CC and cortex of $12 \mathrm{M}$ rTg-DI rats. B: Venn diagram comparing significantly reduced proteins by $\geq 30 \%$ of the wild-type concentration in the $\mathrm{CC}$ and cortex of $12 \mathrm{M} \mathrm{rTg}$-DI rats. C: Heat map depicting relative expression of most enhanced proteins in the CC only (top), both CC and cortex (middle), and cortex only (bottom), along with the corresponding expression in the other region, with red indicating enhanced expression, green representing reduced expression, and color intensity representing the degree of expression change. $\mathbf{D}$ : Heat map depicting relative expression of most reduced proteins arranged as in $\mathbf{C}$ with color shading as in $\mathbf{C} . n=5,6(\mathbf{A}$ and $\mathbf{B}) . P<0.05(\mathbf{A}$ and $\mathbf{B})$. Anxa11, annexin A11; other abbreviations are as indicated in Figure 3.

included Mbp, Plp1, Mag, neurofilament light polypeptide, neurofilament medium polypeptide, and neurofilament heavy chain. As discussed in Decreased Proteins in $12 \mathrm{M}$ and $4 M r T g-D I$ Rat $C C$, down-regulation of these proteins can be an indication of neurodegeneration, demyelination, and damaged axonal integrity. In addition, myelinoligodendrocyte glycoprotein was decreased in the cortex by $41 \%$, and, although it did not meet the effect cutoff, it was reduced $23 \%$ in the CC. Myelin-oligodendrocyte glycoprotein is also important for myelination and axonal integrity, ${ }^{36}$ and thus its reduced expression has implications similar to the other commonly down-regulated proteins.

\section{IPA Upstream Regulators and Causal Networks}

Bioinformatics analysis was performed with IPA to provide disease function and pathway context to the proteomic changes. Differentially expressed proteins from the rTg-DI 
$\mathrm{CC}$ were analyzed both individually and comparatively with those from the cortex. Proteins with $\geq 50 \%$ increases or $\geq 30 \%$ decreases over WT expression were included. Based on directional differential expression of downstream target molecules, IPA predicts activation $(z$ score $>2)$ or inhibition $(z$ score less than -2$)$ states of upstream regulators. ${ }^{37}$ Comparative analysis of the CC and cortex indicated the anti-inflammatory transforming growth factor- $\beta 1$ (TGF- $\beta 1$ ) as activated $(z$ score $=2.065)$ in the $\mathrm{CC}$ but not in the cortex. However, it is worth noting that although they did not meet the threshold for predicted activation ( $\mathrm{z}$ score $>2$ ), many of the affiliated proteins are indeed significantly enhanced in the cortex. A heat map with the relative changes in marker expression in each region is depicted in Figure 5A, with increased expression indicated in red, decreased expression indicated in green, and color intensity based on degree of change from the WT expression.

TGF- $\beta 1$ mRNA expression was recently shown to be significantly enhanced in $12 \mathrm{M}$ rTg-DI rats and IPA analysis of brain regional proteomics identified TGF- $\beta 1$ as an activated upstream regulator in the hippocampus and thalamus of $12 \mathrm{M} \mathrm{rTg-DI}$ rats, ${ }^{10,12}$ consistent with the current findings. Notable among these proteins is HspB1. HspB1 can reportedly inhibit induced endothelial to mesenchymal transition, and preserve endothelial cell integrity. ${ }^{38}$ Treatment with TGF- $\beta 1$ has been reported to enhance HspB1 mRNA expression in astrocytes in vitro. ${ }^{39}$ Furthermore, astrocytes release $\mathrm{HspB} 1$ when treated with $\mathrm{A} \beta$ in vitro. ${ }^{40}$ Thus, TGF- $\beta 1$-regulated HspB1 may be an important component of the astrocyte-mediated inflammatory response in the $\mathrm{CC}$, while also serving protective functions against degeneration of the BBB.

A "causal network analysis" was conducted within IPA that connects differentially expressed downstream target proteins via direct and indirect relationships to predict activation states of causative networks. ${ }^{37}$ It revealed significant activation $(z$ score $=4.158)$ of the thrombin $(F 2)$ network in the $\mathrm{CC}$ but not the cortex (Figure 5B). Twentyeight downstream target molecules in the F2 causal network were differentially expressed in the $\mathrm{CC}$, only seven of which were altered in the cortex. IPA activates the F2 network specifically in the thalamus of $12 \mathrm{M}$ rTg-DI rats, a region that uniquely exhibits numerous thrombotic events, including small vessel occlusions and microbleeds. ${ }^{12}$ None of these events has been observed in the CC of rTg-DI rats (data not shown). However, thrombin has also been reported to induce neurodegeneration after activation of astrocytes, microglia, and neuroinflammatory responses. ${ }^{41-45}$ Thus,
A

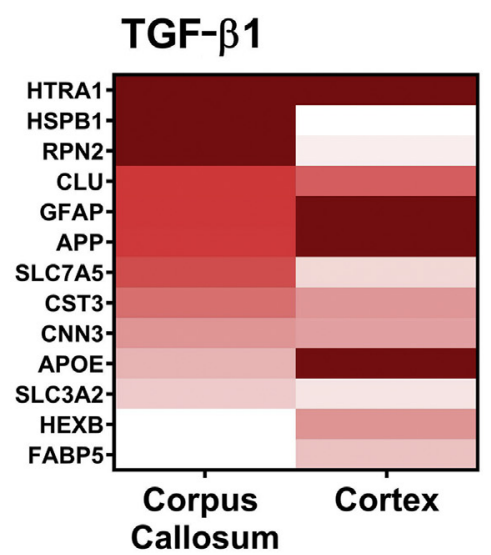

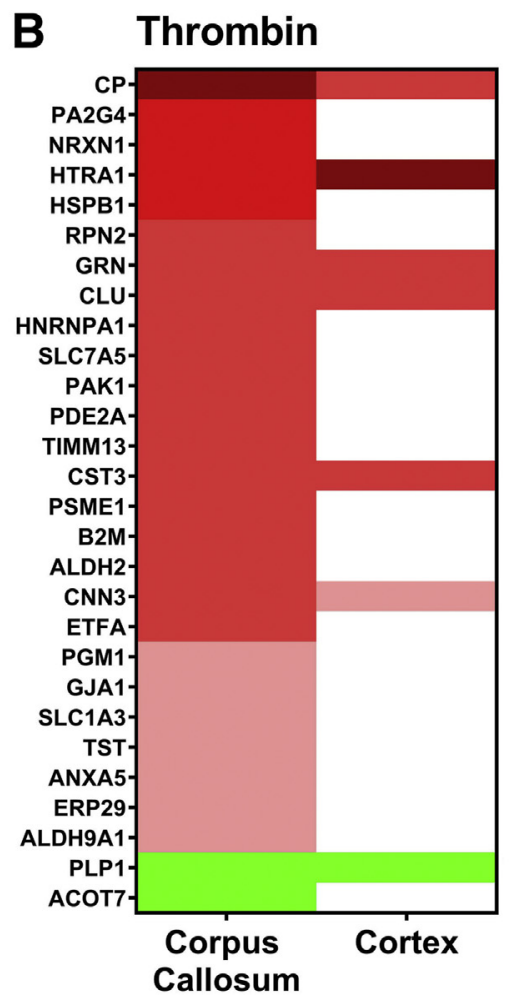

C

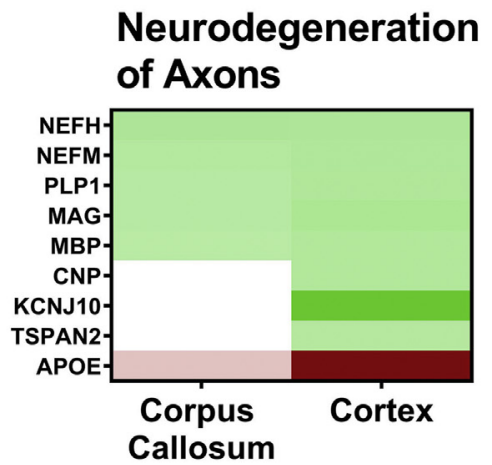

Figure 5 Ingenuity Pathway Analysis (IPA) identified upstream regulators, causal networks, and disease functions. A: Heat map depicting the differentially expressed proteins ( $\geq 50 \%$ increase or $\geq 30 \%$ decrease) in the corpus callosum (CC) and cortex of rTg-DI rats at 12 months (12M) associated with the upstream regulator Tgf- $\beta 1$. B: Heat map depicting the differentially expressed proteins in the CC and cortex of $12 \mathrm{M}$ rTg-DI rats associated with the F2/thrombin causal network. C: Heat map depicting the differentially expressed proteins in the CC and cortex of $12 \mathrm{M}$ rTg-DI rats associated with the disease function Neurodegeneration of Axons. Red indicates increased, green indicates decreased, and white indicates not differentially expressed proteins; color intensity correlates with degree of change. $P<0.05(\mathbf{A})$. Anxa5, annexin A5; other abbreviations are as indicated in Figure 3. 
thrombin signaling could mediate activation of microglia and astrocytes in the rTg-DI CC and induce neuroinflammatory responses that contribute to the degeneration of WM observed in this region.

\section{Downstream Effects Analysis of IPA}

IPA also identifies specific processes and functions causally affected by differentially expressed proteins via downstream effects analysis. IPA provides activation scores for diseases and functions when causal reference information is available. ${ }^{37}$ Comparative downstream effects analysis revealed that neurodegeneration of axons was enhanced in both the $\mathrm{CC}$ and cortex, with activation $z$ scores of 2.217 and 2.783, respectively. It is important to note that although IPA did not include Mbp in the network, it was appropriate to add it in the analysis, as loss of Mbp reportedly can lead to axon demyelination. Additionally, Mbp expression is reduced in the CC of rTg-DI rats exhibiting dramatic WM loss. ${ }^{13,28}$ Relative expression compared with WT in the CC and cortex was mostly similar for the WM-associated proteins Plp1, Mag, and Mbp (Figure 5C). The considerable losses in Plp1, Mbp, and Mag, all with reported roles in myelination and axon integrity, could therefore contribute to the dramatic WM loss characterized in the rTg-DI CC.

\section{Glial Cell Densities in the Cortex and CC}

In rTg-DI rats, the presence of cerebral microvascular amyloid drives strong neuroinflammation, as evidenced by a marked elevation of reactive perivascular glial cells. ${ }^{10,12}$ Many of the differentially expressed proteins in this study can be expressed in both astrocytes and microglia. Therefore, the impact of astrocyte and microglia numbers on the proteomic changes observed in the CC was investigated. In the cortex or CC of $12 \mathrm{M}$ WT rats, there was a normal distribution of astrocytes (Figure 6, A and B). In contrast, in the presence of abundant cortical CAA in the rTg-DI rats, there was a large increase in perivascular activated astrocytes (Figure 6, C and E). However, although the CC of rTg-DI rats lacks appreciable microvascular CAA (Figure 2), there was a highly significant increase $(P<0.001)$ in astrocyte numbers in this region (Figure 6, D and $\mathrm{E})$.

Similar observations were made in the evaluation of activated microglia. In the presence of cortical microvascular CAA, there was a striking increase in the number and activation of perivascular microglia (Figure 7, A-E). Again, in the CC of rTg-DI rats, with small amounts of microvascular amyloid, there was a strong elevation of microglia (Figure 7, D and E). Together, these findings indicate that even with minimal presence of microvascular amyloid, there are robust perivascular glial cell responses that accompany the WM degeneration which occurs in the CC.

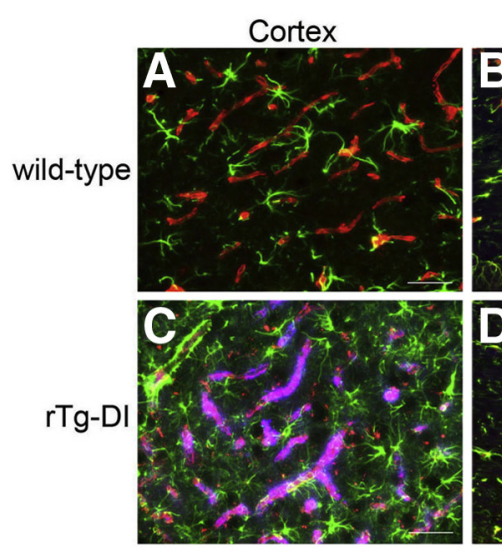

Corpus Callosum
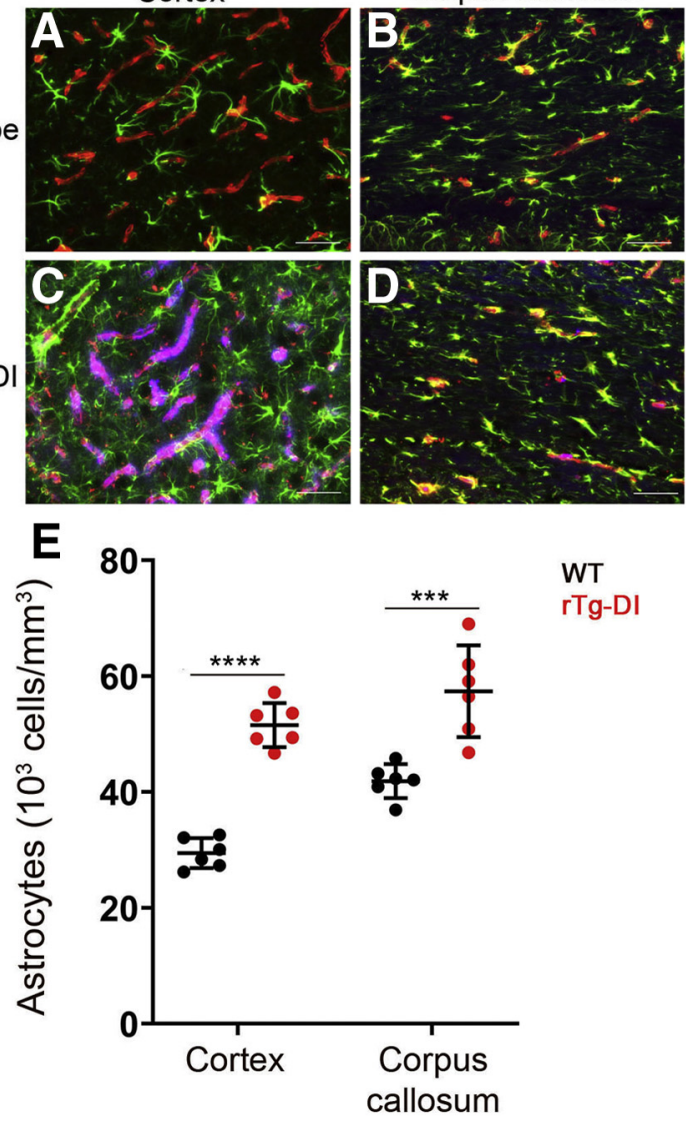

Figure 6 Increased astrocytes in the cortex and corpus callosum (CC) of rTg-DI rats. Brain sections from wild-type (WT) rats (A and $\mathbf{B}$ ) and rTg-DI rats (C and $\mathbf{D}$ ) at 12 months were labeled with Amylo-Glo to detect microvascular fibrillar amyloid (blue), goat polyclonal antibody to collagen IV to detect cerebral microvessels (red), and rabbit polyclonal antibody to glial fibrillary acidic protein to detect astrocytes (green). E: Quantitation of astrocyte numbers from rTg-DI rats (red circles) and WT rats (black circles) at 12 months in the cortex and CC. Data points show the results from each rat and the group mean \pm SD of $n=6 \mathrm{rTg}$-DI rats and $n=6$ WT rats. Compared with WT rats, the astrocyte numbers were significantly elevated in the cortex and CC of the rTg-DI rats. ${ }^{* *} P<0.001 ;{ }^{* * * *} P<0.0001$. Scale bars $=50 \mu \mathrm{m}(\mathbf{A}-\mathbf{D})$.

\section{Validation of Proteomic Analysis and Regional Marker Distribution}

To gain further insight into the cellular responses associated with these proteomic changes, regional immunolabeling was conducted for selected proteins of interest. Anxa3 and specific expression in microglia is enhanced in various brain regions of $12 \mathrm{M}$ rTg-DI rats. ${ }^{12}$ Here, despite minimal CAA burden (Figure 2) and lower microglia density (Figure 7) in the CC compared with the cortex, proteomic results indicated higher total protein concentration (Supplemental Tables S1 and S3) and greater increase in Anxa3 (Figure $4 \mathrm{C}$ ) in the $\mathrm{CC}$ than in the cortex. Interestingly, Anxa3 displayed strong co-localization with the microglia marker Ibal in both the cortex and CC (Figure 8, A-C), indicating its expression in microglia. After normalizing the 


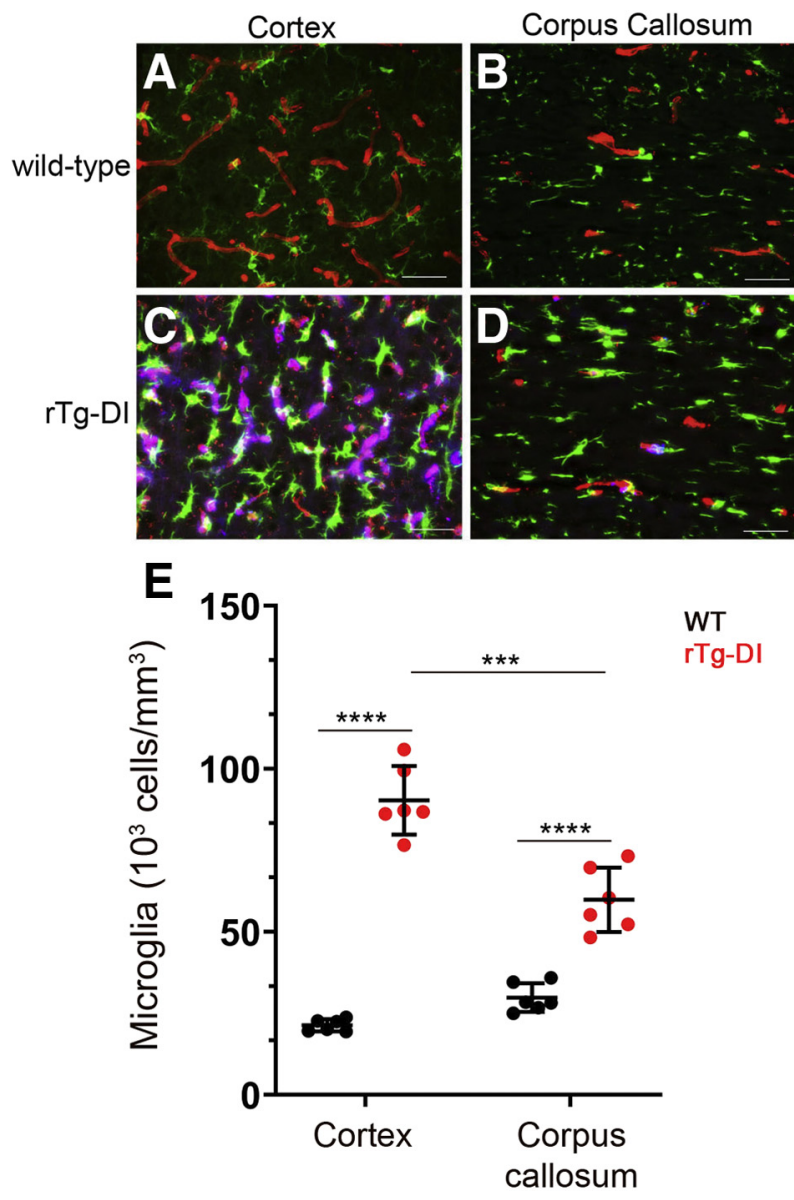

Figure 7 Increased microglia in the cortex and corpus callosum (CC) of rTg-DI rats. Brain sections from wild-type (WT) rats (A and $\mathbf{B}$ ) and rTg-DI rats (C and D) at 12 months were labeled with Amylo-Glo to detect microvascular fibrillar amyloid (blue), rabbit polyclonal antibody to collagen IV to detect cerebral microvessels (red), and goat polyclonal antibody ionized calcium-binding adapter molecule 1 to identify microglia (green). E: Quantitation at 12 months of microglia in rTg-DI rats (red circles) and WT rats (black circles) in the cortex and CC. Data points show the results from each rat and the group mean \pm SD of $n=6 \mathrm{rTg}-\mathrm{DI}$ rats and $n=6$ WT rats. Compared with WT rats, the microglia were significantly elevated in the cortex and $\mathrm{CC}$ of $\mathrm{rTg}$-DI rats. Microglia were elevated more in the cortex compared with the $C C$ of rTg-DI rats. ${ }^{* * *} P<0.001 ;{ }^{* * * *} P<0.0001$. Scale bars $=50 \mu \mathrm{m}(\mathbf{A}-\mathbf{D})$.

total protein concentration (picomoles per milligram total protein) by the microglia density $\left(10^{3}\right.$ cells $\left./ \mathrm{mm}^{3}\right)$, Anxa3 levels per microglia number in the $\mathrm{CC}$ were nearly double that in the cortex. This finding suggests that the more robust increase in Anxa3 in the CC is not merely a function of microgliosis in the presence of vascular amyloid but likely a specific microglial response to WM degeneration.

Annexin A5 (Anxa5), whose expression was recently reported in WM-associated and -activated microglia using single-cell RNA-sequencing, ${ }^{46}$ was up-regulated in the CC (Supplemental Table S1) but not in the cortex of rTg-DI rats. Anxa5 has also been reported to prevent atherothrombosis and reverse $A \beta$-induced toxicity in choroid plexus cell cultures. ${ }^{47,48}$ Based on the reported expression and function of Anxa5, its unique elevation in the $\mathrm{CC}$ of
rTg-DI rats, and the suggested activation of thrombin in the CC by IPA, the regional distribution of Anxa5 via immunolabeling (Figure 8, D-F) was investigated further. Consistent with the proteomic results, Anxa5 displayed specific enhancement in the CC of rTg-DI rats but not in the cortex (Figure 8, D and E), while double-labeling revealed its expression in astrocytes rather than microglia (Figure 8F).

IPA indication of the F2 network and diffuse WM loss in the thalamus of $12 \mathrm{M} \mathrm{rTg-DI}$ rats, ${ }^{12,13}$ both share characteristics in the $\mathrm{CC}$, but in no other investigated regions in the rTg-DI rats. However, the $\mathrm{CC}$ does not share extensive CAA load (Figure 2), nor occluded small vessels/microbleeds with the thalamus. It is possible that based on the reported functions of Anxa5, in the context of atherothrombosis and $A \beta$ toxicity, it harbors protective effects in the $\mathrm{CC}$.

Another protein of interest is MLC1, which displayed dramatic increases $(>400 \%$ over WT) in the rTg-DI CC and only modest changes $(<50 \%$ over WT) in the cortex (Figure 4B). Missense mutations in the $M L C l$ gene lead to the development of MLC, a leukodystrophy characterized by myelin vacuolation, brain edema, and macrocephaly. ${ }^{49}$ Although these phenotypic changes are different from the diffuse WM loss observed in rTg-DI rats, the distinct association of MLC1 with leukodystrophy prompted further investigation of the regional distribution of MLC1. Consistent with the SWATH-MS analysis, immunolabeling revealed extensive up-regulation of MLC1 in the CC, but not in the cortex of rTg-DI rats (Figure 8, G and H). MLC1 is reportedly expressed specifically in astrocytes, ${ }^{49-51}$ and double-immunolabeling with GFAP confirmed strong astrocytic localization in the CC of rTg-DI rats (Figure 8I). MLC can also be caused by missense mutations in GlialCAM, a cell adhesion molecule expressed primarily in glial cells but also found in hepatocytes. ${ }^{51}$ Furthermore, GlialCAM and MLC1 bind one another and co-localize in the astrocytic end feet surrounding cerebral blood vessels and astrocyte-astrocyte contacts. ${ }^{51-53}$ Interestingly, proteomic analysis revealed an approximately $40 \%$ increase in GlialCAM expression in the CC of rTg-DI rats, with no change in the cortex (data not shown). Although this increase was below the 50\% threshold cutoff, because of the increase in GlialCAM protein expression and close association with MLC1, its regional distribution was investigated further. Immunolabeling revealed dramatic enhancements of GlialCAM in the CC of rTg-DI rats but not in the cortex, which was co-localized with astrocytic endfeet (Figure 8, J-L).

\section{Discussion}

CAA is a prevalent feature in most patients with Alzheimer disease, a common cause of cerebral small vessel disease in 

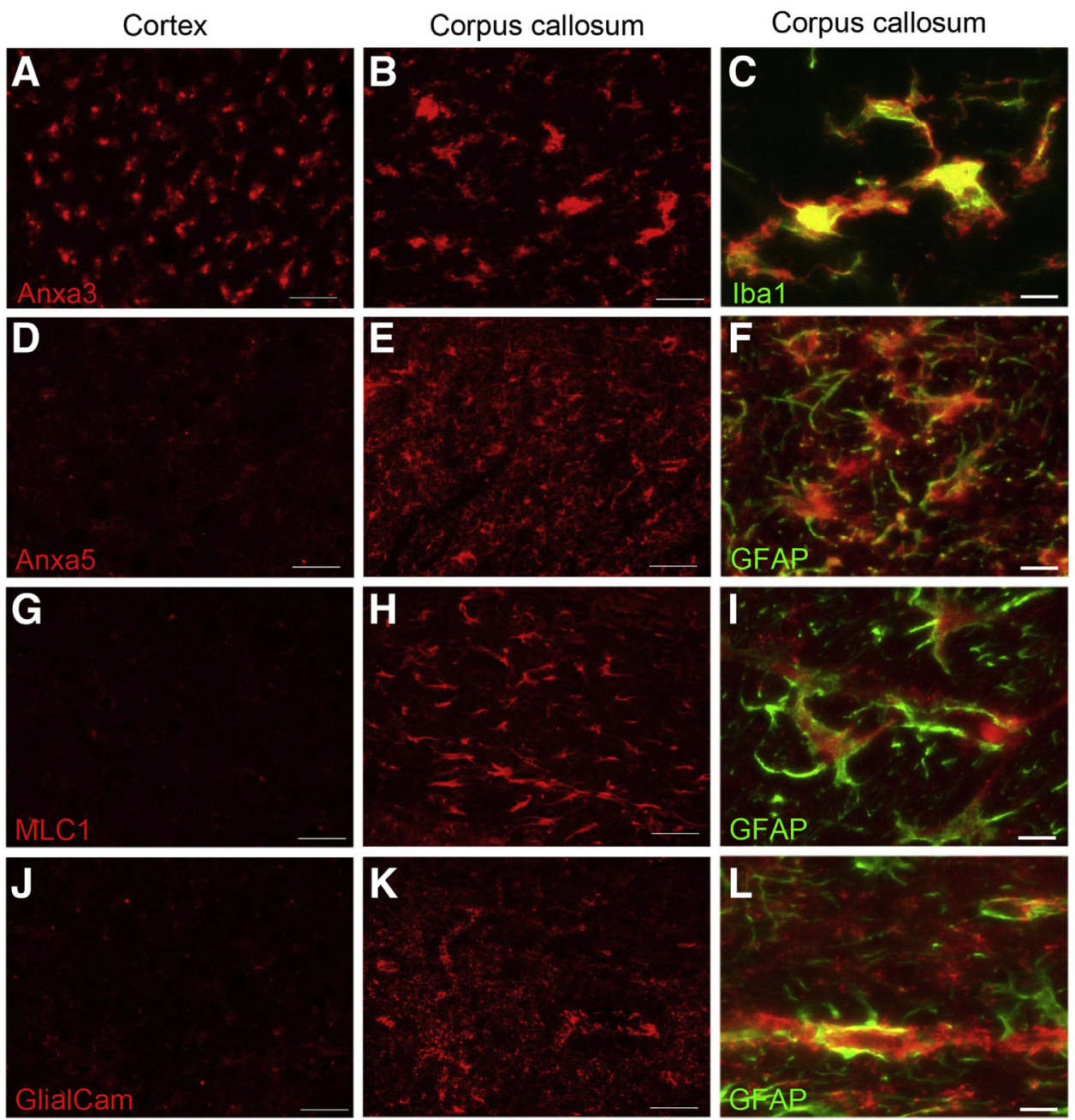

Figure 8 Increased immunolabeling for glial markers in the corpus callosum (CC) of rTg-DI rats. Brain sections at 12 months from the current cohort of rTg-DI rats were immunolabeled with antibodies to annexin A3 (Anxa3) (A-C), annexin A5 (Anxa5) (D-F), megalencephalic leukoencephalopathy with subcortical cysts 1 (MLC1) (G-I), or GlialCAM (J-L) (red) and ionized calcium-binding adapter molecule 1 (Iba-1) (C, green), or glial fibrillary acidic protein (GFAP) (F, I, and L, green). Representative images show increased Anxa3 labeling in microglia in the cortex and CC and increased Anxa5, MLC1, and GlialCAM labeling of astrocytes only in the CC of rTg-DI rats. Scale bars $=50 \mu \mathrm{m}$.

the elderly, and a prominent cause of vascular cognitive impairment and dementia. ${ }^{1-4,6,7}$ Furthermore, the association between CAA and significant diffuse WM loss and WM hypersensitivities has been well documented. ${ }^{13,54,55}$ Although the link between the accumulation of vascular amyloid in the brain and resultant neurodegenerative pathologies remains poorly understood, proteomic analysis can provide further mechanistic insight.

Proteomic approaches in human CAA cases have been reported. ${ }^{2,56,57}$ However, a challenge in investigating human CAA cases is that they originate from diverse backgrounds, including numerous environmental, lifestyle, and genetic differences, thus potentially confounding CAAspecific investigation. Preclinical rodent models provide an advantageous avenue for more uniform population studies, as genetic backgrounds and environmental conditions can be tightly maintained. The rTg-DI model is particularly beneficial because it develops consistent and progressive microvascular CAA, occluded microvessels, and microbleeds. ${ }^{9}$ In addition, rTg-DI rats develop significant perivascular neuroinflammation, disruption of axonal integrity, diffuse WM loss, and behavioral deficits. ${ }^{9-11,13}$ Recently, regional proteomic changes in the cortex, hippocampus, and thalamus of rTg-DI rats were shown to exhibit both similar and distinct differentially expressed proteins reflecting the common and distinguishing pathologic features observed in each region. ${ }^{12}$ Therefore, this approach of regional isolation and SWATH-MS proteomics is an effective method for reporting on specific regional proteomic alterations associated with common and distinct pathologic features of CAA type 1 .

The aim of the current study was to identify proteomic changes within the CC of rTg-DI rats that could provide mechanistic insight into the WM loss associated with CAA type 1 . Moreover, by isolating and analyzing $\mathrm{CC}$ from $4 \mathrm{M}$ to $12 \mathrm{M} \mathrm{rTg-DI}$ rats, progressive proteomic changes in 
animals at early stages of CAA accumulation with no WM loss $(4 \mathrm{M})$ were compared to those with extensive CAA, vascular pathologies, and diffuse WM loss (12M).

Several of the decreased proteins identified at $12 \mathrm{M}$ in rTg-DI CC, including Plp1, Mag, Mbp, neurofilament light polypeptide, neurofilament medium polypeptide, and neurofilament heavy chain, are important for myelination of axons, axonal integrity, and WM homeostasis. Notably, all six of these proteins were also decreased in the cortex (Figure 4D), and neurodegeneration of axons was indicated by IPA analysis in both regions (Figure $5 \mathrm{C}$ ). Although the observed fold decrease of these proteins was similar between the two regions, the concentrations of these proteins ranged from 2.5 to $20 \times$ higher in the CC (Supplemental Table S4). Thus, in terms of total protein, these decreased levels represent much more dramatic changes in the CC versus the cortex, which is consistent with the significant diffuse WM loss observed in the CC (Figure 1). ${ }^{13}$

Activation of thrombin in the $\mathrm{CC}$, indicated by IPA (Figure 5B), could also be a contributing factor to demyelination/neurodegeneration of axons. Although no overt signs of microbleeds or vessel occlusions were observed in the CC (data not shown) thrombin can promote neuroinflammation, astrocytosis, and microgliosis. ${ }^{41-45}$ Recently, it was reported that proteolysis of neurofascin 155, an adhesion protein responsible for the attachment of myelin to axons, is thrombin-dependent and occurs through direct binding of thrombin. ${ }^{58,59}$ In addition, myelin structure/stability can be maintained by paranodal astrocytes through the release of thrombin inhibitors, and blockade of these inhibitors results in demyelination. ${ }^{58}$ Furthermore, deletion of the thrombin receptor protease activated receptor 1 in mice results in enhanced myelination and increased numbers of oligodendrocytes, and blockade of protease activated receptor 1 can promote myelin regeneration in demyelinated lesions. ${ }^{60-62}$ Thus, thrombin could directly contribute to the neurodegeneration, demyelination, and diffuse WM loss observed in the CC and thalamus of rTg-DI rats. ${ }^{13}$

The common enhancement of HTRA1 in the cortex and $\mathrm{CC}$ is also an important observation. Enhancement of HTRA1 has been reported in human CAA cases, ${ }^{22}$ whereas mutations in the HTRA1 gene and loss of HTRA1 function reported causes cerebral autosomal recessive arteriopathy with subcortical infarcts and leukoencephalopathy. ${ }^{32-35}$ Thus, HTRA1 is implicated in both amyloidal and nonamyloidal forms of cerebral small vessel disease associated with leukodystrophies. IPA analysis linked HTRA1 to both the TGF- $\beta 1$ and thrombin networks (Figure 5, A and B). HTRA1 has been reported to regulate TGF- $\beta 1$ signaling, and enhanced HTRA1 mRNA expression can be induced by TGF- $\beta 1 .^{35,63,64}$ Furthermore, dysregulation of TGF- $\beta 1$ signaling as a result of dysfunctional HTRA1 has been suggested as an underlying mechanism of cerebral autosomal recessive arteriopathy with subcortical infarcts and leukoencephalopathy pathologies. ${ }^{35}$ IPA's indicated association of HTRA1 to thrombin is indirect and based on reported links between thrombin and TGF- $\beta 1$. Thrombin can alter TGF- $\beta 1$ signaling in epithelial cells, whereas thrombin injections in the cerebrospinal fluid induced TGF$\beta 1$ pathway-dependent inflammatory responses in rats. ${ }^{65,66}$ Hence, HTRA1 may be an important factor in CAA pathology, possibly related to thrombin and TGF- $\beta 1$ signaling, although its exact role will require further investigation.

Anxa3 was identified as a novel marker for microglia and was implicated in proliferation and migration. ${ }^{67} \mathrm{We}$ recently reported enhanced microglial expression of Anxa3 in several brain regions of $12 \mathrm{M}$ rTg-DI rats. ${ }^{12}$ Here, despite the significantly lower number of microglia in the CC compared with the cortex (Figure 7), Anxa3 expression/microglia number in the $\mathrm{CC}$ was double compared with that in the cortex. Furthermore, Anxa3 was elevated in the $\mathrm{CC}$ of $4 \mathrm{M}$ rTg-DI rats, when CAA burden is minimal (Figure 2). Therefore, Anxa3 may indicate a distinct population of activated microglia in the CC compared with the cortex, with specific roles in areas of WM degeneration that is not affected by CAA burden.

Anxa5 was specifically elevated in the $\mathrm{CC}$ of $12 \mathrm{M}$ rTg-DI rats and not in the cortex (Supplemental Table S2 and Figure 8E). Up-regulation of Anxa5 has been reported in the choroid plexus of patients with Alzheimer disease, ${ }^{68}$ can prevent $A \beta$ aggregation, ${ }^{69,70}$ and has been implicated as an inflammatory marker in $A \beta$-treated microglia. ${ }^{71}$ In the rTg-DI CC, however, Anxa5 expression was observed primarily in astrocytes (Figure 8E). There was no increased Anxa5 expression observed in the cortex, and we did not find Anxa5 up-regulation in other rTg-DI rat brain regions. ${ }^{12}$ Based on its reported direct interactions with $\mathrm{A} \beta$, its specific up-regulation in the $\mathrm{rTg}$-DI CC, and its localization in astrocytes, it is possible that Anxa5 represents a component of a WM-specific astrocyte response to degeneration and CAA pathology. Alternatively, Anxa5 has been reported as a marker of Wallerian degeneration, ${ }^{31}$ and its presence in the CC could indicate occurrence of this process.

The up-regulation of MLC1 and GlialCAM and their relevance to leukodystrophies is also of keen interest. Mutations in the MLC1 and GlialCAM genes are uniquely linked to the incidence of MLC, a leukodystrophy characterized by WM vacuolization, swelling, and brain edema. ${ }^{49}$ MLC1 and GlialCAM bind one another, and GlialCAM serves as a chaperone for membrane localization of MLC1 in astrocytic endfeet contacting blood vessels and other glial cells. ${ }^{49,51,52,72}$ Furthermore, MLC1 and GlialCAM maintain volume-regulated anion currents through interaction with the chloride channel ClC-2. ${ }^{72}$ However, previous studies have solely focused on effects of MLC1 and GlialCAM knockout or missense mutations, and conditions of increased expression have not been reported. Based on their prominent role in other leukodystrophies, it is possible that MLC1 and GlialCAM up-regulation is linked to the diffuse WM loss and myelin degeneration; however, this relationship in the rTg-DI CC requires further investigation. 


\section{Limitations of the Study}

First, the current study focused on proteomic changes that occurred in the CC region of the rTg-DI rat model of microvascular CAA. Because the CC exhibited a combination of WM loss and low levels of CAA, the reported proteomic changes in this specific region could result from a combination of these localized pathologic events. Second, because larger vessel CAA was largely absent in the rTg-DI rats, it is unclear if this form of vascular amyloid may promote different proteomic changes. Third, due to the large number of proteins changed in the $\mathrm{CC}$, only a subset was confirmed by immunohistochemical analysis. Fourth, this study is limited in its ability to report sex-specific proteomic changes due to the small number of female and male animals used. Although no significant differences in protein expression were observed between male and female rTg-DI rats, and their microvascular amyloid loads remained consistent, determination of definitive sex-specific changes in the rTg-DI model will require larger studies with a greater number of male and female rats.

\section{Conclusions}

The current study reveals novel information on proteomic changes that uniquely occur in the CC of rTg-DI rats with emerging WM degeneration. These findings provide new information on potential pathogenic mechanisms that occur in the $\mathrm{CC}$ subsequent to the onset and progression of robust microvascular CAA in adjacent regions of the brain and warrant further systematic investigation in future studies.

\section{Acknowledgments}

We thank Dr. Xiaoyue Zhu and Ms. Judianne Davis for technical assistance.

\section{Author Contributions}

J.M.S., F.X., H.L., and B.B. performed experiments; J.M.S. drafted the manuscript; W.E.V.N. conceptualized and supervised the study; and W.E.V.N. and H.B acquired funding, and reviewed and edited the manuscript.

\section{Supplemental Data}

Supplemental material for this article can be found at http://doi.org/10.1016/j.ajpath.2021.11.010.

\section{References}

1. Attems J, Jellinger K, Thal DR, Van Nostrand W: Review: sporadic cerebral amyloid angiopathy. Neuropathol Appl Neurobiol 2011, 37 : $75-93$
2. Rensink AAM, de Waal RMW, Kremer B, Verbeek MM: Pathogenesis of cerebral amyloid angiopathy. Brain Res Rev 2003, 43: 207-223

3. Arvanitakis Z, Leurgans SE, Wang Z, Wilson RS, Bennett DA, Schneider JA: Cerebral amyloid angiopathy pathology and cognitive domains in older persons. Ann Neurol 2011, 69:320-327

4. Viswanathan A, Greenberg SM: Cerebral amyloid angiopathy in the elderly. Ann Neurol 2011, 70:871-880

5. Thal DR, Ghebremedhin E, Rüb U, Yamaguchi H, Del Tredici K, Braak H: Two types of sporadic cerebral amyloid angiopathy. J Neuropathol Exp Neurol 2002, 61:282-293

6. Boyle PA, Yu L, Nag S, Leurgans S, Wilson RS, Bennett DA Schneider JA: Cerebral amyloid angiopathy and cognitive outcomes in community-based older persons. Neurology 2015, 85:1930-1936

7. Greenberg SM, Vernooij MW, Cordonnier C, Viswanathan A, AlShahi Salman R, Warach S, Launer LJ, Van Buchem MA, Breteler MM; Microbleed Study Group: Cerebral microbleeds: a guide to detection and interpretation. Lancet Neurol 2009, 8:165-174

8. Reijmer YD, van Veluw SJ, Greenberg SM: Ischemic brain injury in cerebral amyloid angiopathy. J Cereb Blood Flow Metab 2016, 36 : $40-54$

9. Davis J, Xu F, Hatfield J, Lee H, Hoos MD, Popescu D, Crooks E, Kim R, Smith SO, Robinson JK, Benveniste H, Van Nostrand WE: A novel transgenic rat model of robust cerebral microvascular amyloid with prominent vasculopathy. Am J Pathol 2018, 188:2877-2889

10. Zhu X, Hatfield J, Sullivan JK, Xu F, Van Nostrand WE: Robust neuroinflammation and perivascular pathology in rTg-DI rats, a novel model of microvascular cerebral amyloid angiopathy. J Neuroinflammation 2020, 17:78

11. Popescu DL, Van Nostrand WE, Robinson JK: Longitudinal cognitive decline in a novel rodent model of cerebral amyloid angiopathy type-1. Int J Mol Sci 2020, 21:2348

12. Schrader JM, Xu F, Nostrand WEV: Distinct brain regional proteome changes in the rTg-DI rat model of cerebral amyloid angiopathy. J Neurochem 2021, 159:273-291

13. Lee H, Xu F, Liu X, Koundal S, Zhu X, Davis J, Yanez D, Schrader J, Stanisavljevic A, Rothman DL, Wardlaw J, Nostrand WEV, Benveniste H: Diffuse white matter loss in a transgenic rat model of cerebral amyloid angiopathy. J Cereb Blood Flow Metab 2020, 41: $1103-1118$

14. Ludwig C, Gillet L, Rosenberger G, Amon S, Collins BC, Aebersold R: Data-independent acquisition-based SWATH-MS for quantitative proteomics: a tutorial. Mol Syst Biol 2018, 14:e8126

15. Aebersold R, Mann M: Mass-spectrometric exploration of proteome structure and function. Nature 2016, 537:347-355

16. López-Sánchez LM, Jiménez-Izquierdo R, Peñarando J, Mena R, Guil-Luna S, Toledano M, Conde F, Villar C, Díaz C, Ortea I, De la Haba-Rodríguez JR, Aranda E, Rodríguez-Ariza A: SWATH-based proteomics reveals processes associated with immune evasion and metastasis in poor prognosis colorectal tumours. J Cell Mol Med 2019, 23:8219-8232

17. Gillet LC, Navarro P, Tate S, Röst H, Selevsek N, Reiter L, Bonner R, Aebersold R: Targeted data extraction of the MS/MS spectra generated by data-independent acquisition: a new concept for consistent and accurate proteome analysis. Mol Cell Proteomics 2012, 11. O111.016717

18. Koundal S, Liu X, Sanggaard S, Mortensen K, Wardlaw J, Nedergaard M, Benveniste H, Lee H: Brain morphometry and longitudinal relaxation time of spontaneously hypertensive rats (SHRs) in early and intermediate stages of hypertension investigated by $3 \mathrm{D}$ VFA-SPGR MRI. Neuroscience 2019, 404:14-26

19. Valdés-Hernández PA, Sumiyoshi A, Nonaka H, Haga R, AubertVásquez E, Ogawa T, Iturria-Medina Y, Riera JJ, Kawashima R: An in vivo MRI template set for morphometry, tissue segmentation, and fMRI localization in rats. Front Neuroinform 2011, 5:26

20. Wiśniewski JR, Rakus D: Multi-enzyme digestion FASP and the 'Total Protein Approach'-based absolute quantification of the Escherichia coli proteome. J Proteomics 2014, 109:322-331 
21. Wiśniewski JR, Ostasiewicz P, Duś K, Zielińska DF, Gnad F, Mann M: Extensive quantitative remodeling of the proteome between normal colon tissue and adenocarcinoma. Mol Syst Biol 2012, 8:611

22. Hondius DC, Eigenhuis KN, Morrema THJ, van der Schors RC, van Nierop P, Bugiani M, Li KW, Hoozemans JJM, Smit AB, Rozemuller AJM: Proteomics analysis identifies new markers associated with capillary cerebral amyloid angiopathy in Alzheimer's disease. Acta Neuropathol Commun 2018, 6:46

23. Pascovici D, Handler DCL, Wu JX, Haynes PA: Multiple testing corrections in quantitative proteomics: a useful but blunt tool. Proteomics 2016, 16:2448-2453

24. Eng LF, Vanderhaeghen JJ, Bignami A, Gerstl B: An acidic protein isolated from fibrous astrocytes. Brain Res 1971, 28:351-354

25. Eng LF, Ghirnikar RS, Lee YL: Glial fibrillary acidic protein: GFAPthirty-one years (1969-2000). Neurochem Res 2000, 25:1439-1451

26. Guttenplan KA, Liddelow SA: Astrocytes and microglia: models and tools. J Exp Med 2019, 216:71-83

27. Liu Q, Xie F, Alvarado-Diaz A, Smith MA, Moreira PI, Zhu X, Perry G: Neurofilamentopathy in neurodegenerative diseases. Open Neurol J 2011, 5:58-62

28. Weil M-T, Möbius W, Winkler A, Ruhwedel T, Wrzos C, Romanelli E, Bennett JL, Enz L, Goebels N, Nave K-A, Kerschensteiner M, Schaeren-Wiemers N, Stadelmann C, Simons M: Loss of myelin basic protein function triggers myelin breakdown in models of demyelinating diseases. Cell Rep 2016, 16:314-322

29. Gould EA, Busquet N, Shepherd D, Dietz RM, Herson PS, Simoes de Souza FM, Li A, George NM, Restrepo D, Macklin WB: Mild myelin disruption elicits early alteration in behavior and proliferation in the subventricular zone. Elife 2018, 7:e34783

30. Pronker MF, Lemstra S, Snijder J, Heck AJR, ThiesWeesie DME, Pasterkamp RJ, Janssen BJC: Structural basis of myelin-associated glycoprotein adhesion and signalling. Nat Commun 2016, 7:13584

31. Sievers C, Platt N, Perry VH, Coleman MP, Conforti L: Neurites undergoing Wallerian degeneration show an apoptotic-like process with annexin $\mathrm{V}$ positive staining and loss of mitochondrial membrane potential. Neurosci Res 2003, 46:161-169

32. Fukutake T: Cerebral autosomal recessive arteriopathy with subcortical infarcts and leukoencephalopathy (CARASIL): from discovery to gene identification. J Stroke Cerebrovasc Dis 2011, 20:85-93

33. Chen Y, He Z, Meng S, Li L, Yang H, Zhang X: A novel mutation of the high-temperature requirement A serine peptidase 1 (HTRA1) gene in a Chinese family with cerebral autosomal recessive arteriopathy with subcortical infarcts and leukoencephalopathy (CARASIL). J Int Med Res 2013, 41:1445-1455

34. Uemura M, Nozaki H, Koyama A, Sakai N, Ando S, Kanazawa M, Kato T, Onodera O: HTRA1 mutations identified in symptomatic carriers have the property of interfering the trimer-dependent activation cascade. [Erratum appeared in Front Neurol 2021, 12:756038.]. Front Neurol 2019, 10:693

35. Beaufort N, Scharrer E, Kremmer E, Lux V, Ehrmann M, Huber R, Houlden H, Werring D, Haffner C, Dichgans M: Cerebral small vessel disease-related protease HTRA1 processes latent TGF-[beta] binding protein 1 and facilitates TGF-[beta] signaling. Proc Natl Acad Sci U S A 2014, 111:16496-16501

36. Peschl P, Bradl M, Höftberger R, Berger T, Reindl M: Myelin oligodendrocyte glycoprotein: deciphering a target in inflammatory demyelinating diseases. Front Immunol 2017, 8:529

37. Krämer A, Green J, Pollard J Jr, Tugendreich S: Causal analysis approaches in ingenuity pathway analysis. Bioinformatics 2014, 30: $523-530$

38. Choi S-H, Nam J-K, Kim B-Y, Jang J, Jin Y-B, Lee H-J, Park S, Ji YH, Cho J, Lee Y-J: HSPB1 inhibits the endothelial-tomesenchymal transition to suppress pulmonary fibrosis and lung tumorigenesis. Cancer Res 2016, 76:1019-1030

39. Hamby ME, Coppola G, Ao Y, Geschwind DH, Khakh BS, Sofroniew MV: Inflammatory mediators alter the astrocyte transcriptome and calcium signaling elicited by multiple G-proteincoupled receptors. J Neurosci 2012, 32:14489-14510

40. Nafar F, Williams JB, Mearow KM: Astrocytes release HspB1 in response to amyloid-[beta] exposure in vitro. J Alzheimers Dis 2016 , 49:251-263

41. Lin C-C, Lee I-T, Wu W-B, Liu C-J, Hsieh H-L, Hsiao L-D, Yang C$\mathrm{C}$, Yang C-M: Thrombin mediates migration of rat brain astrocytes via PLC, $\mathrm{Ca}^{2+}$, CaMKII, PKC[alpha], and AP-1-dependent matrix metalloproteinase-9 expression. Mol Neurobiol 2013, 48:616-630

42. Lee DY, Park KW, Jin BK: Thrombin induces neurodegeneration and microglial activation in the cortex in vivo and in vitro: proteolytic and non-proteolytic actions. Biochem Biophys Res Commun 2006, 346: 727-738

43. Huang C, Ma R, Sun S, Wei G, Fang Y, Liu R, Li G: JAK2-STAT3 signaling pathway mediates thrombin-induced proinflammatory actions of microglia in vitro. J Neuroimmunol 2008, 204:118-125

44. Ye X, Zuo D, Yu L, Zhang L, Tang J, Cui C, Bao L, Zan K, Zhang Z, Yang X, Chen H, Tang H, Zu J, Shi H, Cui G: ROS/TXNIP pathway contributes to thrombin induced NLRP3 inflammasome activation and cell apoptosis in microglia. Biochem Biophys Res Commun 2017, 485:499-505

45. Yin $M$, Chen Z, Ouyang Y, Zhang H, Wan Z, Wang H, Wu W, Yin X: Thrombin-induced, TNFR-dependent miR-181c downregulation promotes MLL1 and NF-[kappa]B target gene expression in human microglia. J Neuroinflammation 2017, 14:132

46. Safaiyan S, Besson-Girard S, Kaya T, Cantuti-Castelvetri L, Liu L, Ji H, Schifferer M, Gouna G, Usifo F, Kannaiyan N, Fitzner D, Xiang X, Rossner MJ, Brendel M, Gokce O, Simons M: White matter aging drives microglial diversity. Neuron 2021, 109: 1100-1117.e10

47. Cederholm A, Frostegård J: Annexin A5 as a novel player in prevention of atherothrombosis in SLE and in the general population. Ann N Y Acad Sci 2007, 1108:96-103

48. Bartolome F, Krzyzanowska A, de la Cueva M, Pascual C, Antequera D, Spuch C, Villarejo-Galende A, Rabano A, Fortea J, Alcolea D, Lleo A, Ferrer I, Hardy J, Abramov AY, Carro E: Annexin A5 prevents amyloid- $\beta$-induced toxicity in choroid plexus: implication for Alzheimer's disease. Sci Rep 2020, 10:9391

49. Hwang J, Vu HM, Kim M-S, Lim H-H: Plasma membrane localization of MLC1 regulates cellular morphology and motility. Mol Brain 2019, 12:116

50. Dubey M, Bugiani M, Ridder MC, Postma NL, Brouwers E, Polder E, Jacobs JG, Baayen JC, Klooster J, Kamermans M, Aardse R, de Kock CPJ, Dekker MP, van Weering JRT, Heine VM, Abbink TEM, Scheper GC, Boor I, Lodder JC, Mansvelder HD, van der Knaap MS: Mice with megalencephalic leukoencephalopathy with cysts: a developmental angle. Ann Neurol 2015, 77:114-131

51. Hoegg-Beiler MB, Sirisi S, Orozco IJ, Ferrer I, Hohensee S, Auberson M, Gödde K, Vilches C, de Heredia ML, Nunes V, Estévez R, Jentsch TJ: Disrupting MLC1 and GlialCAM and ClC-2 interactions in leukodystrophy entails glial chloride channel dysfunction. Nat Commun 2014, 5:3475

52. López-Hernández T, Ridder MC, Montolio M, Capdevila-Nortes X, Polder E, Sirisi S, Duarri A, Schulte U, Fakler B, Nunes V, Scheper GC, Martínez A, Estévez R, van der Knaap MS: Mutant GlialCAM causes megalencephalic leukoencephalopathy with subcortical cysts, benign familial macrocephaly, and macrocephaly with retardation and autism. Am J Hum Genet 2011, 88:422-432

53. López-Hernández T, Sirisi S, Capdevila-Nortes X, Montolio M, Fernández-Dueñas V, Scheper GC, van der Knaap MS, Casquero P, Ciruela F, Ferrer I, Nunes V, Estévez R: Molecular mechanisms of MLC1 and GLIALCAM mutations in megalencephalic leukoencephalopathy with subcortical cysts. Hum Mol Genet 2011, 20: 3266-3277

54. Reijmer YD, Fotiadis P, Charidimou A, van Veluw SJ, Xiong L, Riley GA, Martinez-Ramirez S, Schwab K, Viswanathan A, Gurol ME, Greenberg SM: Relationship between white matter 
connectivity loss and cortical thinning in cerebral amyloid angiopathy. Hum Brain Mapp 2017, 38:3723-3731

55. Fotiadis P, van Rooden S, van der Grond J, Schultz A, MartinezRamirez S, Auriel E, Reijmer Y, van Opstal AM, Ayres A, Schwab KM; Alzheimer's Disease Neuroimaging Initiative, Hedden T, Rosand J, Viswanathan A, Wermer M, Terwindt G, Sperling RA, Polimeni JR, Johnson KA, van Buchem MA, Greenberg SM, Gurol ME: Cortical atrophy in hereditary and sporadic forms of cerebral amyloid angiopathy: an observational casecontrol study. Lancet Neurol 2016, 15:811-819

56. Manousopoulou A, Gatherer M, Smith C, Nicoll JAR, Woelk CH, Johnson M, Kalaria R, Attems J, Garbis SD, Carare RO: Systems proteomic analysis reveals that clusterin and tissue inhibitor of metalloproteinases 3 increase in leptomeningeal arteries affected by cerebral amyloid angiopathy. Neuropathol Appl Neurobiol 2017, 43:492-504

57. Manousopoulou A, Yuen HM, Sharp MM, Saito S, Aldea R, Mazer N, Garbis SD, Carare RO: Quantitative proteomic profiling of white matter in cases of cerebral amyloid angiopathy reveals upregulation of extracellular matrix proteins and clusterin. Free Neuropathol 2020, 1:28

58. Dutta DJ, Woo DH, Lee PR, Pajevic S, Bukalo O, Huffman WC, Wake H, Basser PJ, SheikhBahaei S, Lazarevic V, Smith JC, Fields RD: Regulation of myelin structure and conduction velocity by perinodal astrocytes. Proc Natl Acad Sci U S A 2018, 115:11832-11837

59. Dutta DJ, Fields RD: Deletion of the thrombin proteolytic site in neurofascin 155 causes disruption of nodal and paranodal organization. Front Cell Neurosci 2021, 15:576609

60. Choi C-I, Yoon H, Drucker KL, Langley MR, Kleppe L, Scarisbrick IA: The thrombin receptor restricts subventricular zone neural stem cell expansion and differentiation. Sci Rep 2018, 8:9360

61. Yoon H, Radulovic M, Drucker KL, Wu J, Scarisbrick IA: The thrombin receptor is a critical extracellular switch controlling myelination. Glia 2015, 63:846-859

62. Yoon H, Choi C-I, Triplet EM, Langley MR, Kleppe LS, Kim HN, Simon WL, Scarisbrick IA: Blocking the thrombin receptor promotes repair of demyelinated lesions in the adult brain. J Neurosci 2020, 40: $1483-1500$

63. Friedrich U, Datta S, Schubert T, Plössl K, Schneider M, Grassmann F, Fuchshofer R, Tiefenbach K-J, Längst G, Weber BHF:
Synonymous variants in HTRA1 implicated in AMD susceptibility impair its capacity to regulate TGF-[beta] signaling. Hum Mol Genet 2015, 24:6361-6373

64. Xu L, Golshirazian I, Asbury BJ, Li Y: Induction of high temperature requirement $\mathrm{A} 1$, a serine protease, by TGF-betal in articular chondrocytes of mouse models of OA. Histol Histopathol 2014, 29: 609-618

65. Bae J-S, Kim I-S, Rezaie AR: Thrombin down-regulates the TGFbeta-mediated synthesis of collagen and fibronectin by human proximal tubule epithelial cells through the EPCR-dependent activation of PAR-1. J Cell Physiol 2010, 225:233-239

66. Li T, Zhang P, Yuan B, Zhao D, Chen Y, Zhang X: Thrombininduced TGF- $\beta 1$ pathway: a cause of communicating hydrocephalus post subarachnoid hemorrhage. Int J Mol Med 2013, 31:660-666

67. Zhang Z, Li Z, Ma Z, Deng M, Xing M, Wu J, Jiang S, Wang Q, Guo Q, Zou W: Annexin A3 as a marker protein for microglia in the central nervous system of rats. Neural Plast 2021, 2021:5575090

68. Krzyzanowska A, García-Consuegra I, Pascual C, Antequera D, Ferrer I, Carro E: Expression of regulatory proteins in choroid plexus changes in early stages of Alzheimer disease. J Neuropathol Exp Neurol 2015, 74:359-369

69. Lee G, Pollard HB, Arispe N: Annexin 5 and apolipoprotein E2 protect against Alzheimer's amyloid- $\beta$-peptide cytotoxicity by competitive inhibition at a common phosphatidylserine interaction site. Peptides 2002, 23:1249-1263

70. Hung LW, Ciccotosto GD, Giannakis E, Tew DJ, Perez K, Masters CL, Cappai R, Wade JD, Barnham KJ: Amyloid-beta peptide (Abeta) neurotoxicity is modulated by the rate of peptide aggregation: Abeta dimers and trimers correlate with neurotoxicity. J Neurosci 2008, 28:11950-11958

71. Correani V, Di Francesco L, Mignogna G, Fabrizi C, Leone S, Giorgi A, Passeri A, Casata R, Fumagalli L, Maras B, Schininà ME: Plasma membrane protein profiling in beta-amyloid-treated microglia cell line. Proteomics 2017, 17

72. Capdevila-Nortes X, López-Hernández T, Apaja PM, López de Heredia M, Sirisi S, Callejo G, Arnedo T, Nunes V, Lukacs GL, Gasull X, Estévez R: Insights into MLC pathogenesis: GlialCAM is an MLC1 chaperone required for proper activation of volumeregulated anion currents. Hum Mol Genet 2013, 22:4405-4416 